\title{
Exactly Solvable Schrödinger Operators
}

\author{
Jan Dereziński and Michał Wrochna
}

\begin{abstract}
We systematically describe and classify one-dimensional Schrödinger equations that can be solved in terms of hypergeometric type functions. Beside the well-known families, we explicitly describe two new classes of exactly solvable Schrödinger equations that can be reduced to the Hermite equation.
\end{abstract}

\section{Introduction}

Exactly solvable one-dimensional Schrödinger equations play an important role in quantum physics. The best known are the harmonic oscillator and the radial equation for the hydrogen atom [22], which are covered in every course of quantum mechanics. A number of other examples were discovered in the 1930s of the last century. They include (known also under other names) trigonometric and hyperbolic Pöschl-Teller potentials [19], the Scarf potential [20], the Eckart potential [5], the Manning-Rosen potential [15] and the RosenMorse potential. All these examples can be reduced to the hypergeometric equation (see e.g. [24]). One should also mention the Morse potential that leads to the confluent equation [14]. Problems involving these potentials are often used in classes on quantum mechanics, see e.g. the well-known problem book of Flügge [6]. A number of techniques have been developed to study their properties, such as the factorization method [8] and the closely related method of the superpotential (see for instance $[3,4]$ ).

In later years, Ginocchio [7] discovered that these examples can be generalized to a larger class of potentials equivalent to the hypergeometric or confluent equation. Later, this class was extended by Natanzon [16] and further generalized by Milson [13]. These classes, besides the hitherto known potentials, are not very practical in applications, since they are not given by explicit expressions.

There exist even more general classes of potentials that can be called exactly solvable, found by Khare and Sukhatme [11]. They are, however, expressed in exotic special functions. 
The literature on exactly solvable Schrödinger equations is very large. The subject is in fact very useful for applications, especially in quantum physics. We also believe that it is quite beautiful, which in the existing literature is perhaps not so easy to see.

In our paper, we would like to systematically describe basic classes of exactly solvable Schrödinger equations. Most of the material of our paper is scattered in the literature, notably in $[3,13,16]$ (see also [1]). Our treatment is, however, somewhat more systematic than what we could find in the literature. For instance, we distinguish between the complex classification and the real classification. It also seems that for the first time we explicitly describe two new classes of exactly solvable Schrödinger equations, which can be reduced to the Hermite equation (see Sects. 5.2 and 5.3).

By a (stationary one-dimensional) Schrödinger equation, we will mean an equation of the form

$$
\left(-\partial_{r}^{2}+V(r)-E\right) \phi(r)=0 .
$$

This equation can be interpreted as the eigenvalue problem for the operator

$$
H:=-\partial_{r}^{2}+V(r) .
$$

An operator of the form (1.2) will be called a (one-dimensional) Schrödinger operator. $V(r)$ will be called a potential and the parameter $E$ an energy.

Equation (1.2) can be interpreted as an operator in a number of ways. If $V$ is a holomorphic function of the complex variable on some open $\Omega \subset \mathbb{C}$, then $r$ can be interpreted as a complex variable and (1.2) can be viewed as an operator on holomorphic functions. In this case, it is natural to allow $E$ to be a complex parameter. The corresponding eigenvalue equation (1.1) will be then called the Schrödinger equation of the complex variable. Let us note that, complex affine transformations preserve the class of Schrödinger equations of the complex variable. (By a complex affine transformations, we mean $r \mapsto a r+b$, where $a \neq 0, b$ are complex constants).

One can also interpret $r$ as a real variable in some open $I \subset \mathbb{R}$. The operator (1.2) is then viewed as acting on functions on $I$. Understood in this way, (1.1) will be called the Schrödinger equation of the real variable. Of special interest is then the case of real potentials. Clearly, real affine transformations preserve the class of real Schrödinger equations of the real variable.

Our paper is organized as follows. First, we will briefly discuss some general facts related to second-order linear differential equations. In particular, we will describe basic ingredients of the so-called Bose-Natanzon method, which permits to obtain a class of Schrödinger equations equivalent to a chosen linear equation. Note that it applies to more general situations than those described in the literature $[13,16]$. We will formulate a criterion that determines when the Bose-Natanzon method can be used.

Next, we will focus on hypergeometric type equations. Recall that equations of the form

$$
\left(\sigma(z) \partial_{z}^{2}+\tau(z) \partial_{z}+\eta\right) f(z)=0
$$


where $\sigma(z), \tau(z)$ are polynomials with

$$
\operatorname{deg} \sigma \leq 2, \quad \operatorname{deg} \tau \leq 1,
$$

and $\eta$ is a number, are sometimes called hypergeometric type equations (see Nikiforov and Uvarov [17]). Solutions of (1.3) are called hypergeometric type functions. Hypergeometric type functions are very well understood and include the Gauss hypergeometric function, the confluent function, Hermite, Laguerre and Jacobi polynomials, etc. Traditionally, one classifies hypergeometric type equations into several distinct classes invariant under complex affine transformations. In each class, one chooses a simple representative, to which the whole class can be reduced. Such representatives are the hypergeometric equation, the confluent equation, etc.

In our analysis, we will concentrate on three specific equations:

(1) the hypergeometric equation

$$
\left(z(1-z) \partial_{z}^{2}+(c-(a+b+1) z) \partial_{z}-a b\right) f(z)=0
$$

(2) the rescaled confluent equation

$$
\left(z \partial_{z}^{2}+(c-\gamma z) \partial_{z}-a\right) f(z)=0
$$

(3) the translated harmonic oscillator

$$
\left(-\partial_{z}^{2}+\theta^{2} z^{2}+\rho z+\lambda\right) f(z)=0 .
$$

Note that all hypergeometric type equations with a nonzero $\sigma$ are related to one of the above equations by elementary operations

- multiplication of $f(z)$ by a function, multiplication of the equation by a function,

- a change of variables not depending on the parameters $a, b, c$ (respectively, $a, c, \gamma$ or $\theta, \rho, \lambda)$.

(1) If $\sigma$ is second order and has two distinct roots, an affine transformation reduces (1.3) to (1.4).

(2) If $\sigma$ is second order and has only one root, then a transformation involving $z \mapsto z^{-1}$ reduces (1.3) to (1.5) with $\gamma \neq 0$.

(3) If $\sigma$ is first order, then an affine transformation reduces (1.3) to(1.5).

(4) If $\sigma$ is zeroth order and nonzero, then (1.3) is equivalent to (1.6) with $\theta \neq 0$.

Obviously, (1.4) and (1.5) are themselves equations of the hypergeometric type. (1.6) is not, but can be reduced to a hypergeometric type equation. This reduction depends on whether $\theta \neq 0$ or $\theta=0$. The former case leads to a hypergeometric type equation with a constant $\sigma$, as mentioned above. In the case $\theta=0,(1.6)$ is the Airy equation, which can be reduced to a special case of the hypergeometric type equation by a transformation involving $z \mapsto z^{3}$.

Equations (1.4), (1.5) and (1.6) are very well understood and have well known solutions. We will describe classes of solvable potentials reducible to one of Eqs. (1.4), (1.5) or (1.6). We will describe both the complex and the real classification of such potentials. 
It is natural to consider first a classification of exactly solvable Schrödinger equations of the complex variable. Note that all solvable potentials that we consider are holomorphic on the complex plane, apart from some isolated singularities. Obviously, we can always use a complex affine transformation to put the equation in a convenient form. One can also move a complex constant from the potential to the energy.

Consider a family of exactly solvable holomorphic potentials. Suppose that for a selected subfamily of parameters the potential is real if restricted to an open, possibly infinite interval $I \subset \mathbb{C}$. By elementary properties of holomorphic functions, if we extend the interval $I$, the potential is still real until we hit a singularity. A real affine transformation can be used to put the equation in a convenient form. In particular, we can always assume that $I$ is a subset of the real line. We can also move a real constant from the potential to the energy.

The above discussion motivates the following definition: We will say that an open interval $I \subset \mathbb{R}$ is a natural real domain for a certain family of potentials if it ends either at $-\infty,+\infty$ or at a singularity of the potential, and no singularities lie inside $I$. When describing the real classification, we will always specify a natural real domain of the potential.

Clearly, if $I$ is a natural real domain, then the operator $H=-\partial_{r}^{2}+V(r)$ is hermitian on $C_{\mathrm{c}}^{\infty}(I)$. It is then natural to ask about self-adjoint extensions of $H$. We will not discuss this question here. We plan to consider it in later papers.

In Eqs. (1.4), (1.5) and (1.6), we have three arbitrary parameters. Therefore, in all the solvable cases that we will describe, the potential depends on two parameters, since the third parameter is responsible for the energy $E$.

\section{Second-Order Homogeneous Differential Equations}

The main objects of our paper are ordinary homogeneous second-order linear differential equations, i.e. equations of the form

$$
\left(a(r) \partial_{r}^{2}+b(r) \partial_{r}+c(r)\right) \phi(r)=0 .
$$

It will be convenient to treat (2.7) as the problem of finding the kernel of the operator

$$
\mathcal{A}\left(r, \partial_{r}\right):=a(r) \partial_{r}^{2}+b(r) \partial_{r}+c(r) .
$$

We will then say that Eq. (2.7) is given by the operator (2.8).

We will treat $r$ either as a complex or a real variable. In the complex case we will usually assume that the coefficients are analytic.

In this section, we describe some general facts related to the theory of equations of the form (2.7) and their reduction to the Schrödinger equation.

\subsection{The Bose Invariant of a Second-Order Differential Equation}

Suppose that we are given a second-order differential equation (2.7). Then we can always eliminate the first-order term as follows. We divide from the left 
by $a(r)$, set

$$
h(r):=\exp \left(\int^{r} \frac{b(t)}{2 a(t)} \mathrm{d} t\right)
$$

We check that

$$
a(r)^{-1} h(r) \mathcal{A}\left(r, \partial_{r}\right) h(r)^{-1}=\partial_{r}^{2}+I(r),
$$

where, using the notation $a^{\prime}:=\frac{\mathrm{d}}{\mathrm{d} r} a(r)$,

$$
I=\frac{4 a c-2 a b^{\prime}+2 b a^{\prime}-b^{2}}{4 a^{2}} .
$$

Clearly, $\check{\phi}(r):=h(r) \phi(r)$ solves the equation

$$
\left(\partial_{r}^{2}+I(r)\right) \check{\phi}(r)=0 .
$$

Equation (2.10) will be called the canonical form of Eq. (2.7). Equation (2.9) will be called the Bose invariant of (2.7) [1]. (The name "Bose invariant" is used e.g. by Milson [13]; however, the object itself was clearly known before Bose).

It is easy to check that for any functions $f, g$ the equation

$$
f(r) \mathcal{A}\left(r, \partial_{r}\right) g(r) \phi(r)=0
$$

has the same Bose invariant as (2.7). Conversely, if an equation has the same Bose invariant as (2.7), it equals (2.11) for some $f, g$.

\subsection{Schwarz Derivative}

The Schwarz derivative of $r \mapsto r(y)$ appears naturally in the context of second order ordinary differential equations and was known to 19th century mathematicians [21]. It was mentioned already by Kummer in 1836 [10]. The universally adopted name Schwarz derivative was introduced by Cayley, who derived its basic properties in 1880 [2]. For more historical comments together with numerous applications, we refer the reader to a book by Osgood [18].

The Schwarz derivative is defined as

$$
\{r, y\}=\left(\frac{r^{\prime \prime}(y)}{r^{\prime}(y)}\right)^{\prime}-\frac{1}{2}\left(\frac{r^{\prime \prime}(y)}{r^{\prime}(y)}\right)^{2} .
$$

Here are some basic properties of the Schwarz derivative:

Proposition 2.1. 1. Let $y \mapsto s(y), s \mapsto r(s)$ be two functions. Then

$$
\{r, y\}=\{r, s\} s^{\prime}(y)^{2}+\{s, y\} .
$$

2. Let $r \mapsto y(r)$ be the inverse of $y \mapsto r(y)$. Then

$$
\{y, r\}=-\left[r^{\prime}(y)\right]^{-2}\{r, y\} .
$$

3. $\{r, y\} \equiv 0 \Leftrightarrow r(y)=\frac{a y+b}{c y+d}, a d-b c \neq 0$.

We will need the following fact: 
Proposition 2.2. Suppose that $z^{\prime}=z^{p}(1-z)^{q}$. Then

$$
\{z, r\}=\frac{1}{2} z^{2 p-2}(1-z)^{2 q-2}\left(\left(p^{2}-2 p\right)(1-z)^{2}+\left(q^{2}-2 q\right) z^{2}-2 p q z(1-z)\right) .
$$

\subsection{The Liouville Transformation}

Consider a second-order equation in the canonical form, that is

$$
\left(\partial_{r}^{2}+I(r)\right) \phi(r)=0 .
$$

Let us make the transformation $r=r(y)$ in this equation. We obtain

$$
\frac{1}{\left[r^{\prime}(y)\right]^{2}} \partial_{y}^{2} \phi(r(y))-\frac{r^{\prime \prime}(y)}{\left[r^{\prime}(y)\right]^{3}} \partial_{y} \phi(r(y))+I(r(y)) \phi(r(y))=0 .
$$

The resulting equation we transform again to its canonical form:

$$
\left(\partial_{y}^{2}+J(y)\right) \psi(y)=0
$$

where

$$
\begin{aligned}
\psi(y) & =\frac{1}{\left[r^{\prime}(y)\right]^{2}} \phi(r(y)), \\
J(y) & =\left[r^{\prime}(y)\right]^{2} I(r(y))+\frac{1}{2}\{r, y\} .
\end{aligned}
$$

The above procedure is called the Liouville transformation of (2.16) by the change of variables $r=r(y)$ [12]. One can check using (2.17) that the composition of two Liouville transformations, first by the change of variables $r=$ $r(y)$, then $y=y(z)$, is the Liouville transformation by the change of variables $r=r(y(z))$.

\subsection{Natanzon's Problem}

Let $\mathcal{A}\left(a_{1}, \ldots, a_{k} ; z, \partial_{z}\right)$ be a second-order differential operator depending on $k$ parameters, such that its Bose invariant can be written as

$$
I\left(b_{1}, \ldots, b_{n} ; z\right)=b_{1} I_{1}(z)+\cdots+b_{n} I_{n}(z)
$$

for some (at least one) $n \leq k$ linearly independent functions $I_{1}, \ldots, I_{n}$ (independent of $\left.a_{1}, \ldots, a_{k}\right)$, and some $n$ numbers $b_{i}\left(a_{1}, \ldots, a_{k}\right)$. Then one can solve the following problem (which we will call Natanzon's problem for $\mathcal{A}\left(z, \partial_{z}\right)$ ):

Find all potentials $V(r)$ such that the 1-dimensional stationary Schrödinger equation

$$
\left(-\partial_{r}^{2}+V(r)-E\right) \phi(E, r)=0
$$

can be transformed to the equation given by $\mathcal{A}\left(a_{1}, \ldots, a_{k} ; z, \partial_{z}\right)$ for some $a_{1}(E), \ldots, a_{k}(E)$. We allow the following operations:

(1) multiplication of both sides of the equation by some $f(E, r)$;

(2) substitution of $\check{\phi}(E, r):=g(E, r) \phi(E, r)$ for some $g(E, r)$;

(3) change of coordinates $r \mapsto z$ independent of $E$. 
This is the problem solved by Natanzon in the case of the hypergeometric equation and by Milson in the general case of hypergeometric type equations. In the following, we recall the construction they used, in a slightly generalized form.

Let us consider an arbitrary equation of the form (2.22). Obviously, its Bose invariant equals $E-V(r)$. Clearly, the transformations (1) and (2) allow us to transform $\mathcal{A}\left(a_{1}, \ldots, a_{k} ; z, \partial_{z}\right)$ to its canonical form

$$
-\partial_{z}^{2}-I\left(b_{1}, \ldots, b_{n} ; z\right) \text {. }
$$

Thus (2.22) can be transformed to $\mathcal{A}\left(a_{1}, \ldots, a_{k} ; z, \partial_{z}\right)$ if

$$
I\left(b_{1}, \ldots, b_{n} ; z\right)=\left(r^{\prime}(z)\right)^{2}\left(E-V(r(z))+\frac{1}{2}\{r, z\},\right.
$$

i.e., if the two Bose invariants are related by a Liouville transformation. Using (2.21) we rewrite $(2.24)$ as

$$
b_{1}(E) I_{1}(z)+\cdots+b_{n}(E) I_{n}(z)=\left(r^{\prime}(z)\right)^{2}\left(E-V(r(z))+\frac{1}{2}\{r, z\} .\right.
$$

By assumption, $b_{i}$ depend on $E$ and $I_{i}(z)$ are independent of $E$. Thus, the dependence of $b_{i}$ on $E$ is linear. Therefore, by transforming linearly $\left(b_{1}, \ldots, b_{n}\right)$ into $\left(\tilde{b}_{1}, \ldots, \tilde{b}_{n-1}, E\right)$, we can assume that

$$
I\left(b_{1}, \ldots, b_{n} ; z\right)=\tilde{b}_{1} \tilde{I}_{1}(z)+\cdots+\tilde{b}_{n-1} \tilde{I}_{n-1}(z)+E \tilde{I}_{n}(z),
$$

for some functions $\tilde{I}_{i}(z)$, which are linear combinations of $I_{i}(z)$. Then we can write

$$
E \tilde{I}_{n}(z)+\tilde{b}_{1} \tilde{I}_{1}(z)+\cdots+\tilde{b}_{n-1} \tilde{I}_{n-1}(z)=\left(r^{\prime}(z)\right)^{2}\left(E-V(r(z))+\frac{1}{2}\{r, z\} .\right.
$$

Therefore, Natanzon's problem is solved by the following pair of equations

$$
\begin{aligned}
V\left(\tilde{b}_{1}, \ldots, \tilde{b}_{n-1}, r(z)\right) & :=\left(r^{\prime}(z)\right)^{-2}\left(\tilde{b}_{1} \tilde{I}_{1}(z)+\cdots+\tilde{b}_{n-1} \tilde{I}_{n-1}(z)-\frac{1}{2}\{r, z\}\right), \\
\left(r^{\prime}(z)\right)^{2} & =\tilde{I}_{n}(z) .
\end{aligned}
$$

We can rewrite (2.27) in an equivalent way as

$$
\begin{aligned}
V\left(\tilde{b}_{1}, \ldots, \tilde{b}_{n-1}, r\right) & :=\left(z^{\prime}(r)\right)^{2}\left(\tilde{b}_{1} \tilde{I}_{1}(z(r))+\cdots+\tilde{b}_{n-1} \tilde{I}_{n-1}(z(r))\right)+\frac{1}{2}\{z, r\}, \\
\left(z^{\prime}(r)\right)^{-2} & =\tilde{I}_{n}(z(r)) .
\end{aligned}
$$

If desired, we can renormalize $E$ by subtracting a constant.

Note that in practical applications the value of the above method depends on whether it is possible to invert the relation $r \mapsto z$ expressing it in terms of standard functions. In the next sections, we will describe such solutions of the Natanzon problem for the three classes of equations described in Sect. 1. 


\section{Schrödinger Operators Reducible to the Hypergeometric Equation}

\subsection{Hypergeometric Equation}

The hypergeometric equation is given by the operator

$$
\mathcal{F}\left(a, b ; c ; z, \partial_{z}\right):=z(1-z) \partial_{z}^{2}+(c-(a+b+1) z) \partial_{z}-a b,
$$

where $a, b, c$ are arbitrary complex parameters. Its Bose invariant equals

$$
{ }_{2} I_{1}(a, b ; c ; z)=\frac{\left(1-a^{2}-b^{2}-2 a b\right) z^{2}+2(-2 a b+a c+b c-c) z+2 c-c^{2}}{4 z^{2}(1-z)^{2}} .
$$

It satisfies condition (2.21) for $n=3$. The functions $I_{i}(z), i=1,2,3$ can be taken to be $\frac{1}{4 z^{2}(1-z)} \frac{1}{4 z(1-z)^{2}}$ and $\frac{1}{4 z(1-z)}$. It is natural to demand that $\left(r^{\prime}(z)\right)^{2}=\tilde{I}_{3}(z)$ is a function of the form $z^{2 p}(1-z)^{2 q}$. It is easy to see that this gives the following possibilities for $\tilde{I}_{3}(z)$ :

$$
\begin{aligned}
& \text { (1) } \frac{1}{4 z(1-z)}, \quad \text { (2) } \frac{1}{4 z^{2}(1-z)}, \quad \text { (3) } \frac{1}{4 z(1-z)^{2}}, \\
& \text { (4) } \frac{1}{4 z^{2}(1-z)^{2}}, \quad \text { (5) } \frac{1}{4 z^{2}}, \quad \text { (6) } \frac{1}{4(1-z)^{2}} .
\end{aligned}
$$

We will see that each of these Ansatzes leads to exactly solvable potentials considered in the literature. Note also that the formula for the Schwarz derivative (2.15) will be handy.

\subsection{The Riemann Equation}

It is well known that it is useful to consider the hypergeometric equation as a special case of the so-called Riemann equation. The Riemann equation is defined as the class of equations on the Riemann sphere $\overline{\mathbb{C}}$ having three regular singular points. The following theorem summarizes the basic theory of these equations:

Theorem 3.1. (1) Suppose that we are given a second-order differential equation on the Riemann sphere having three singular points $z_{1}, z_{2}, z_{3}$, all of them regular singular points with the following indices

$$
\begin{array}{ll}
z_{1}: & \rho_{1}, \tilde{\rho}_{1}, \\
z_{2}: & \rho_{2}, \tilde{\rho}_{2}, \\
z_{3}: & \rho_{3}, \tilde{\rho}_{3} .
\end{array}
$$

Then the following condition is satisfied:

$$
\rho_{1}+\tilde{\rho}_{1}+\rho_{2}+\tilde{\rho}_{2}+\rho_{3}+\tilde{\rho}_{3}=1 .
$$

Such an equation, normalized to have coefficient 1 at the second derivative, is always equal to

$$
\mathcal{P}\left(\begin{array}{cccc}
z_{1} & z_{2} & z_{3} & \\
\rho_{1} & \rho_{2} & \rho_{3} & z, \partial_{z} \\
\tilde{\rho}_{1} & \tilde{\rho}_{2} & \tilde{\rho}_{3} &
\end{array}\right) \phi(z)=0
$$


where

$$
\begin{aligned}
\mathcal{P}\left(\begin{array}{cccc}
z_{1} & z_{2} & z_{3} & \\
\rho_{1} & \rho_{2} & \rho_{3} & z, \partial_{z} \\
\tilde{\rho}_{1} & \tilde{\rho}_{2} & \tilde{\rho}_{3}
\end{array}\right):= & \partial_{z}^{2}-\left(\frac{\rho_{1}+\tilde{\rho}_{1}-1}{z-z_{1}}+\frac{\rho_{2}+\tilde{\rho}_{2}-1}{z-z_{2}}+\frac{\rho_{3}+\tilde{\rho}_{3}-1}{z-z_{3}}\right) \partial_{z} \\
& +\frac{\rho_{1} \tilde{\rho}_{1}\left(z_{1}-z_{2}\right)\left(z_{1}-z_{3}\right)}{\left(z-z_{1}\right)^{2}\left(z-z_{2}\right)\left(z-z_{3}\right)}+\frac{\rho_{2} \tilde{\rho}_{2}\left(z_{2}-z_{3}\right)\left(z_{2}-z_{1}\right)}{\left(z-z_{2}\right)^{2}\left(z-z_{3}\right)\left(z-z_{1}\right)} \\
& +\frac{\rho_{3} \tilde{\rho}_{3}\left(z_{3}-z_{1}\right)\left(z_{3}-z_{2}\right)}{\left(z-z_{3}\right)^{2}\left(z-z_{1}\right)\left(z-z_{2}\right)}
\end{aligned}
$$

(2) Let $z \mapsto w=h(z)=\frac{a z+b}{c z+d}$. (Transformations of this form are called homographies or Möbius transformations). We can always assume that $a d-b c=1$. Then

$$
\mathcal{P}\left(\begin{array}{cccc}
h\left(z_{1}\right) & h\left(z_{2}\right) & h\left(z_{3}\right) & \\
\rho_{1} & \rho_{2} & \rho_{3} & w, \partial_{w} \\
\tilde{\rho}_{1} & \tilde{\rho}_{2} & \tilde{\rho}_{3} &
\end{array}\right)=(c z+d)^{4} \mathcal{P}\left(\begin{array}{cccc}
z_{1} & z_{2} & z_{3} & \\
\rho_{1} & \rho_{2} & \rho_{3} & z, \partial_{z} \\
\tilde{\rho}_{1} & \tilde{\rho}_{2} & \tilde{\rho}_{3} &
\end{array}\right)
$$

$$
\begin{aligned}
& \left.\left(z-z_{1}\right)^{-\lambda}\left(z-z_{2}\right)^{\lambda} \mathcal{P}\left(\begin{array}{ccc}
z_{1} & z_{2} & z_{3} \\
\rho_{1} & \rho_{2} & \rho_{3} \\
\tilde{\rho}_{1} & \tilde{\rho}_{2} & \tilde{\rho}_{3}
\end{array}\right], \partial_{z}\right)\left(z-z_{1}\right)^{\lambda}\left(z-z_{2}\right)^{-\lambda} \\
& \quad=\mathcal{P}\left(\begin{array}{cccc}
z_{1} & z_{2} & z_{3} & \\
\rho_{1}-\lambda & \rho_{2}+\lambda & \rho_{3} & z, \partial_{z} \\
\tilde{\rho}_{1}-\lambda & \tilde{\rho}_{2}+\lambda & \tilde{\rho}_{3} &
\end{array}\right)
\end{aligned}
$$

Clearly, in all above formulas one of $z_{i}$ can equal $\infty$, with an obvious meaning of various expressions. For convenience we give the expression for the Riemann operator with $z_{3}=\infty$ :

$$
\begin{aligned}
\mathcal{P}\left(\begin{array}{cccc}
z_{1} & z_{2} & \infty & \\
\rho_{1} & \rho_{2} & \rho_{3} & z, \partial_{z} \\
\tilde{\rho}_{1} & \tilde{\rho}_{2} & \tilde{\rho}_{3} &
\end{array}\right) \\
=\partial_{z}^{2}-\left(\frac{\rho_{1}+\tilde{\rho}_{1}-1}{z-z_{1}}+\frac{\rho_{2}+\tilde{\rho}_{2}-1}{z-z_{2}}\right) \partial_{z} \\
\quad+\frac{\rho_{1} \tilde{\rho}_{1}\left(z_{1}-z_{2}\right)}{\left(z-z_{1}\right)^{2}\left(z-z_{2}\right)}+\frac{\rho_{2} \tilde{\rho}_{2}\left(z_{2}-z_{1}\right)}{\left(z-z_{2}\right)^{2}\left(z-z_{1}\right)}+\frac{\rho_{3} \tilde{\rho}_{3}}{\left(z-z_{1}\right)\left(z-z_{2}\right)} .
\end{aligned}
$$

The hypergeometric equation is a special case of the Riemann equation, since

$$
\begin{aligned}
& \mathcal{F}\left(a, b ; c ; z, \partial_{z}\right)=z(1-z) \mathcal{P}\left(\begin{array}{cccc}
0 & 1 & \infty & \\
0 & 0 & a & z, \partial_{z} \\
1-c & c-a-b & b &
\end{array}\right) \\
& =z(1-z) \partial_{z}^{2}+(c-(a+b+1) z) \partial_{z}-a b .
\end{aligned}
$$

Every Riemann equation can be brought to the form of the hypergeometric equation by applying (1) and (2) of Theorem 3.1. 
From Theorem 3.1, we also see that symmetries of the hypergeometric equation are better visible if we replace $a, b, c$ with $\alpha, \beta, \mu$ :

$$
\begin{gathered}
\alpha=c-1, \quad \beta=a+b-c, \quad \mu=a-b ; \\
a=\frac{1+\alpha+\beta-\mu}{2}, \quad b=\frac{1+\alpha+\beta+\mu}{2}, \quad c=1+\alpha .
\end{gathered}
$$

In fact, the new parameters coincide with the differences of the indices of the points $0,1, \infty$ :

$$
\alpha=\rho_{1}-\tilde{\rho}_{1}, \quad \beta=\rho_{2}-\tilde{\rho}_{2}, \quad \mu=\rho_{3}-\tilde{\rho}_{3} .
$$

In the parameters $\alpha, \beta, \mu$, the Bose invariant of the hypergeometric equation has a more symmetric form:

$$
{ }_{2} I_{1}(\alpha, \beta, \mu ; z)=\frac{\left(1-\alpha^{2}\right)(1-z)+\left(1-\beta^{2}\right) z+\left(\mu^{2}-1\right)(1-z) z}{4 z^{2}(1-z)^{2}} .
$$

We can summarize the relation between the hypergeometric equation and its canonical form by

$$
\begin{aligned}
& -z^{\frac{\alpha}{2}+\frac{1}{2}-1}(1-z)^{\frac{\beta}{2}+\frac{1}{2}-1} \mathcal{F}\left(\frac{\alpha+\beta+\mu+1}{2}, \frac{\alpha+\beta-\mu+1}{2} ; 1+\alpha ; z, \partial_{z}\right) \\
& \times z^{-\frac{\alpha}{2}-\frac{1}{2}}(1-z)^{-\frac{\beta}{2}-\frac{1}{2}} \\
& =-\mathcal{P}\left(\begin{array}{cccc}
0 & 1 & \infty & \\
\frac{\alpha}{2}+\frac{1}{2} & \frac{\beta}{2}+\frac{1}{2} & \frac{\mu}{2}-\frac{1}{2} & z, \partial_{z} \\
-\frac{\alpha}{2}+\frac{1}{2} & -\frac{\beta}{2}+\frac{1}{2} & -\frac{\mu}{2}-\frac{1}{2} &
\end{array}\right) \\
& =-\partial_{z}^{2}+\left(\alpha^{2}-1\right) \frac{1}{4 z^{2}(1-z)}+\left(\beta^{2}-1\right) \frac{1}{4 z(1-z)^{2}}-\left(\mu^{2}-1\right) \frac{1}{4 z(1-z)} \text {. }
\end{aligned}
$$

It will be natural to introduce the parameters

$$
\kappa:=\frac{1}{2}\left(\alpha^{2}-\beta^{2}\right), \quad \delta:=\frac{1}{2}\left(\alpha^{2}+\beta^{2}\right)
$$

In some cases the parameter $\kappa$ will be replaced by

$$
\tau:=\frac{\mathrm{i}}{2}\left(\alpha^{2}-\beta^{2}\right)=\mathrm{i} \kappa .
$$

We will describe two complex classes of exactly solvable potentials depending on two complex parameters. Within each complex class there will be three real classes of exactly solvable potentials depending on two real parameters.

\subsection{Trigonometric Pöschl-Teller Potential}

In this subsection we consider Ansatz (1) of (3.30). We set

$$
z=\sin ^{2} \frac{r}{2}, \quad \text { which solves } z^{\prime}=-(-z)^{\frac{1}{2}}(1-z)^{\frac{1}{2}} .
$$

This leads to the Schrödinger equation

$$
\left(-\partial_{r}^{2}+V_{\delta, \kappa}^{\mathrm{tPT}}(r)-\frac{\mu^{2}}{4}\right) \phi(r)=0,
$$


where

$$
\begin{aligned}
V_{\delta, \kappa}^{\mathrm{tPT}}(r) & :=\left(\alpha^{2}-\frac{1}{4}\right) \frac{1}{4 \sin ^{2} \frac{r}{2}}+\left(\beta^{2}-\frac{1}{4}\right) \frac{1}{4 \cos ^{2} \frac{r}{2}} \\
& =\left(\delta-\frac{1}{4}\right) \frac{1}{\sin ^{2} r}+\kappa \frac{\cos r}{\sin ^{2} r}
\end{aligned}
$$

This potential was proposed and solved by Pöschl and Teller [19]. It is usually called the Pöschl-Teller potential, sometimes also the Pöschl-Teller potential of the first kind or the trigonometric Scarf potential.

A natural real domain for this potential is $] 0, \pi[$. If $\kappa, \delta$ are real, then the potential is real on this domain.

Explicitly, the reduction of (3.38) to the hypergeometric equation is derived as follows:

$$
\begin{aligned}
& -z^{\frac{\alpha}{2}+\frac{1}{4}}(1-z)^{\frac{\beta}{2}+\frac{1}{4}} \mathcal{F}\left(\frac{\alpha+\beta+\mu+1}{2}, \frac{\alpha+\beta-\mu+1}{2} ; 1+\alpha ; z, \partial_{z}\right) z^{-\frac{\alpha}{2}-\frac{1}{4}}(1-z)^{-\frac{\beta}{2}-\frac{1}{4}} \\
& =-z(1-z) \mathcal{P}\left(\begin{array}{cccc}
0 & 1 & \infty & \\
\frac{\alpha}{2}+\frac{1}{4} & \frac{\beta}{2}+\frac{1}{4} & \frac{\mu}{2} & z, \partial_{z} \\
-\frac{\alpha}{2}+\frac{1}{4} & -\frac{\beta}{2}+\frac{1}{4} & -\frac{\mu}{2} &
\end{array}\right) \\
& =-z(1-z)\left(\partial_{z}^{2}+\left(\frac{1}{2 z}-\frac{1}{2(1-z)}\right) \partial_{z}\right) \\
& +\left(\alpha^{2}-\frac{1}{4}\right) \frac{1}{4 z}+\left(\beta^{2}-\frac{1}{4}\right) \frac{1}{4(1-z)}-\frac{\mu^{2}}{4} \\
& -\partial_{r}^{2}+\left(\alpha^{2}-\frac{1}{4}\right) \frac{1}{4 \sin ^{2} \frac{r}{2}}+\left(\beta^{2}-\frac{1}{4}\right) \frac{1}{4 \cos ^{2} \frac{r}{2}}-\frac{\mu^{2}}{4} .
\end{aligned}
$$

\subsection{Hyperbolic Pöschl-Teller Potential}

We continue with (1) of (3.30). We set

$$
z=-\sinh ^{2} \frac{r}{2}, \quad \text { which solves } z^{\prime}=-(-z)^{\frac{1}{2}}(1-z)^{\frac{1}{2}} .
$$

This leads to the Schrödinger equation

$$
\left(-\partial_{r}^{2}+V_{\delta, \kappa}^{\mathrm{hPT}}(r)+\frac{\mu^{2}}{4}\right) \phi(r)=0,
$$

where

$$
\begin{aligned}
V_{\delta, \kappa}^{\mathrm{hPT}}(r) & :=\left(\alpha^{2}-\frac{1}{4}\right) \frac{1}{4 \sinh ^{2} \frac{r}{2}}-\left(\beta^{2}-\frac{1}{4}\right) \frac{1}{4 \cosh ^{2} \frac{r}{2}} \\
& =\left(\delta-\frac{1}{4}\right) \frac{1}{\sinh ^{2} r}+\kappa \frac{\cosh r}{\sinh ^{2} r} .
\end{aligned}
$$

This potential was also proposed and solved by Pöschl and Teller [19]. In the literature it is known as hyperbolic, generalized Pöschl-Teller potential, or the Pöschl-Teller potential of the second kind.

A natural real domain for this potential is $] 0, \infty[$. If $\kappa, \delta$ are real, then the potential is real on this domain. 
To see that (3.42) can be solved in terms of the hypergeometric equation, we first repeat the computations leading to (3.40), and then set $z=-\sinh ^{2} \frac{r}{2}$.

\subsection{Scarf Potential}

We continue with (1) of (3.30). We set

$$
z=\frac{1}{2}-\mathrm{i} \cosh \frac{r}{2} \sinh \frac{r}{2}, \quad \text { which solves } z^{\prime}=(-z)^{\frac{1}{2}}(1-z)^{\frac{1}{2}} .
$$

This leads to the Schrödinger equation

$$
\left(-\partial_{r}^{2}+V_{\delta, \tau}^{\mathrm{S}}(r)+\frac{\mu^{2}}{4}\right) \phi(r)=0
$$

where

$$
V_{\delta, \tau}^{\mathrm{S}}(r):=-\left(\delta-\frac{1}{4}\right) \frac{1}{\cosh ^{2} r}-\tau \frac{\sinh r}{\cosh ^{2} r} .
$$

This potential was proposed and solved by Scarf [20]. In the literature it is often called the hyperbolic Scarf potential.

A natural real domain for this potential is ] $-\infty, \infty[$. If $\delta, \tau$ are real, then the potential is real on this domain.

To see that (3.45) can be solved in terms of the hypergeometric equation, we first repeat the computations leading to $(3.40)$, and then set $z=$ $\frac{1}{2}-\mathrm{i} \cosh \frac{r}{2} \sinh \frac{r}{2}$.

\subsection{More about Pöschl-Teller and Scarf Potentials}

Both kinds of Pöschl-Teller potentials and the Scarf potential are real cases of the same complex case. To see this, consider e.g. the hyperbolic Pöschl-Teller potential as a function of the complex parameter $r$. It is holomorphic away from singularities at $\mathrm{i} \pi n, n \in \mathbb{Z}$.

For real $\delta, \kappa, V_{\delta, \kappa}^{\mathrm{hPT}}(r)$ is real on $\mathrm{i} \mathbb{R}$ and $\mathbb{R}+\mathrm{i} \pi n$. On each halfline ] $0, \infty[+\mathrm{i} \pi n$ and $]-\infty, 0[+\mathrm{i} \pi n$ we obtain the hyperbolic Pöschl-Teller potential. On each interval ] $\mathrm{i} \pi n, \mathrm{i} \pi(n+1)$ [ we obtain the trigonometric Pöschl-Teller potential.

For real $\delta, \mathrm{i} \kappa, V_{\delta, \kappa}^{\mathrm{hPT}}(r)$ is real on $\mathbb{R}+\mathrm{i} \pi\left(n+\frac{1}{2}\right)$. On each of these lines we obtain the Scarf potential.

Above, we used Ansatz (1) to derive Scarf and both kinds of PöschlTeller potentials. Alternatively, one can use Ansatzes (2) or (3). To see this it is enough to consider Ansatz (3). In fact, we first repeat computations analogous to $(3.40)$ :

$$
\begin{aligned}
& -z(1-z)^{2} \mathcal{P}\left(\begin{array}{ccc}
0 & 1 & \infty \\
\frac{\alpha}{2}+\frac{1}{4} & \frac{\beta}{2} & \left.\frac{\mu}{2}+\frac{1}{4} \quad z, \partial_{z}\right) \\
-\frac{\alpha}{2}+\frac{1}{4} & -\frac{\beta}{2} & -\frac{\mu}{2}+\frac{1}{4}
\end{array}\right) \\
& =-z(1-z)^{2}\left(\partial_{z}^{2}+\left(\frac{1}{2 z}-\frac{1}{1-z}\right) \partial_{z}\right) \\
& \quad+\left(\alpha^{2}-\frac{1}{4}\right) \frac{1-z}{4 z}+\frac{\beta^{2}}{4}-\left(\mu^{2}-\frac{1}{4}\right) \frac{(1-z)}{4}
\end{aligned}
$$


We set

$$
z=\operatorname{tgh}^{2} \frac{r}{2}, \quad \text { which solves } z^{\prime}=z^{\frac{1}{2}}(1-z),
$$

obtaining the hyperbolic Pöschl-Teller potential,

$$
z=-\operatorname{tg}^{2} \frac{r}{2}, \quad \text { which solves } z^{\prime}=-(-z)^{\frac{1}{2}}(1-z),
$$

obtaining the trigonometric Pöschl-Teller potential, or

$$
z=\operatorname{ctgh}^{2} \frac{r}{2}, \quad \text { which solves } z^{\prime}=z^{\frac{1}{2}}(1-z),
$$

obtaining the Scarf potential.

\subsection{Manning-Rosen Potential}

Let us consider Ansatz (4) of (3.30). We set

$$
z=\frac{1}{1+\mathrm{e}^{2 r}}, \quad \text { which solves } z^{\prime}=2 z(z-1) .
$$

This leads to the Schrödinger equation

$$
\left(-\partial_{r}^{2}+V_{\kappa, \mu}^{\mathrm{MR}}(r)+\delta\right) \phi(r)=0
$$

where

$$
V_{\kappa, \mu}^{\mathrm{MR}}(r):=-\kappa \frac{\sinh r}{\cosh r}-\left(\frac{\mu^{2}}{4}-\frac{1}{4}\right) \frac{1}{\cosh ^{2} r} .
$$

This potential was proposed and solved by Manning and Rosen [15]. In the literature it is also called the Woods-Saxon potential [23] (for instance in [6]), also the hyperbolic Rosen-Morse potential.

A natural real domain for this potential is ] $-\infty, \infty[$. The potential is real if $r \in]-\infty, \infty\left[\right.$ and $\kappa, \beta^{2}$ are real.

Here is a derivation of (3.50) from the hypergeometric equation:

$$
\begin{aligned}
& -4 z^{1+\frac{\alpha}{2}}(1-z)^{1+\frac{\beta}{2}} \mathcal{F}\left(\frac{\alpha+\beta+\mu+1}{2}, \frac{\alpha+\beta-\mu+1}{2} ; 1+\alpha ; z, \partial_{z}\right) z^{-\frac{\alpha}{2}}(1-z)^{-\frac{\beta}{2}} \\
& -4 z^{2}(z-1)^{2} \mathcal{P}\left(\begin{array}{cccc}
0 & 1 & \infty & \\
\frac{\alpha}{2} & \frac{\beta}{2} & \mu+\frac{1}{2} & z, \partial_{z} \\
-\frac{\alpha}{2} & -\frac{\beta}{2} & -\mu+\frac{1}{2} &
\end{array}\right) \\
& =-4 z^{2}(1-z)^{2}\left(\partial_{z}^{2}+\left(\frac{1}{z}-\frac{1}{1-z}\right) \partial_{z}\right)+\alpha^{2}(1-z)+\beta^{2} z-\left(\mu^{2}-1\right) z(1-z) \\
& =-\partial_{r}^{2}+\delta+\kappa \frac{\mathrm{e}^{2 r}-1}{1+\mathrm{e}^{2 r}}-\left(\mu^{2}-1\right) \frac{\mathrm{e}^{2 r}}{\left(1+\mathrm{e}^{2 r}\right)^{2}} .
\end{aligned}
$$




\subsection{Eckart Potential}

We still consider Ansatz (4) of (3.30).

We set

$$
z=\frac{1}{1-\mathrm{e}^{-2 r}}, \quad \text { which solves } z^{\prime}=2 z(1-z),
$$

and use (3.35). This leads to the Schrödinger equation

$$
\left(-\partial_{r}^{2}+V_{\kappa, \mu}^{\mathrm{E}}(r)+\delta\right) \phi(r)=0,
$$

where

$$
V_{\kappa, \mu}^{\mathrm{E}}(y):=-\kappa \frac{\cosh r}{\sinh r}+\left(\frac{\mu^{2}}{4}-\frac{1}{4}\right) \frac{1}{\sinh ^{2} r} .
$$

This potential was proposed and solved by Eckart [5]. In the literature (for instance [6]) it is also called the Hulthen potential [9], sometimes also the generalized Morse potential, because of its similarity to the Morse potential, see Sect. 4.4.

A natural real domain for this potential is $] 0, \infty\left[\right.$. If $\kappa, \beta^{2}$ are real, then the potential is real on this domain.

To derive the Eckart potential, we first repeat the computations leading to (3.51), and then set $z=\frac{1}{1-\mathrm{e}^{-2 r}}$.

\subsection{Rosen-Morse Potential}

Once again, we consider Ansatz (4) of (3.30).

We set

$$
z=\frac{1}{1-\mathrm{e}^{2 \mathrm{i} r}}, \quad \text { which solves } z^{\prime}=2 \mathrm{i} z(1-z) .
$$

This leads to the Schrödinger equation

$$
\left(-\partial_{r}^{2}+V_{\tau, \mu}^{\mathrm{RM}}(r)-\delta\right) \phi(r)=0,
$$

where

$$
V_{\tau, \mu}^{\mathrm{RM}}(y):=\tau \frac{\cos r}{\sin r}+\left(\frac{\mu^{2}}{4}-\frac{1}{4}\right) \frac{1}{\sin ^{2} r} .
$$

This potential is known as the Rosen-Morse potential, also the trigonometric Rosen-Morse potential (although this name is widely used in the literature, we were unable to explain decisively its origin).

A natural real domain for this potential is $] 0, \pi\left[\right.$. If $\tau, \mu^{2}$ are real, then the potential is real on this domain.

To derive the Rosen-Morse potential, we first repeat the computations leading to (3.51), then set $z=\frac{1}{1-\mathrm{e}^{2 \mathrm{ir} r}}$.

\subsection{More About Manning-Rosen, Eckart and Rosen-Morse Potentials}

The Manning-Rosen, Eckart and Rosen-Morse potentials are all real cases of the same complex case. To see this, consider e.g. the Eckart potential as a function of the complex parameter $r$. It is holomorphic away from singularities at $\mathrm{i} \pi n, n \in \mathbb{Z}$. 
For real $\kappa, \mu^{2}, V_{\kappa, \mu}^{\mathrm{E}}(r)$ is real on the lines $\mathbb{R}+\frac{\mathrm{i} \pi n}{2}$. On each line $\mathbb{R}+$ $\mathrm{i}\left(m+\frac{1}{2}\right) \pi, m \in \mathbb{Z}$, we obtain the Manning-Rosen potential. On each halfline ] $0, \infty[+\mathrm{i} \pi m$ and $]-\infty, 0[+\mathrm{i} \pi m$, we obtain the Eckart potential.

For real $\mathrm{i} \kappa, \mu^{2}, V_{\kappa, \mu}^{\mathrm{E}}(r)$ is real on $\mathrm{i} \mathbb{R}$. On each interval $] \mathrm{i} \pi m, \mathrm{i} \pi(m+1)[$ we obtain the Rosen-Morse potential.

Ansatzes (5) and (6) lead to the same classes of exactly solvable potentials as Ansatz (4). To see this it is enough to consider Ansatz (5). In fact, we first repeat computations analogous to (3.51):

$$
\begin{aligned}
& -4 z^{-\frac{\alpha+1}{2}}(1-z)^{2+\frac{\beta}{2}} \mathcal{F}\left(\frac{\alpha+\beta+\mu+1}{2}, \frac{\alpha+\beta+-\mu+1}{2} ; 1+\alpha ; z, \partial_{z}\right) z^{-\frac{\alpha+1}{2}}(1-z)^{-\frac{\beta}{2}} \\
& =-4(1-z)^{2} \mathcal{P}\left(\begin{array}{cccc}
0 & 1 & \infty & \\
\alpha+\frac{1}{2} & \frac{\beta}{2} & \frac{\mu}{2} & z, \partial_{z} \\
-\alpha+\frac{1}{2} & -\frac{\beta}{2} & -\frac{\mu}{2} &
\end{array}\right) \\
& =-4(1-z)^{2}\left(\partial_{z}^{2}-\frac{1}{1-z} \partial_{z}\right)+\left(\alpha^{2}-1\right) \frac{1-z}{z^{2}}+\beta^{2} \frac{1}{z}-\mu^{2} \frac{1-z}{z} .
\end{aligned}
$$

We set

$$
z=1-\mathrm{e}^{2 r}, \quad \text { which solves } z^{\prime}=-2(1-z),
$$

obtaining the Eckart potential,

$$
z=1+\mathrm{e}^{2 r}, \quad \text { which solves } z^{\prime}=2(1-z),
$$

obtaining the Manning-Rosen potential,

$$
z=1-\mathrm{e}^{\mathrm{i} 2 r}, \quad \text { which solves } z^{\prime}=-2 \mathrm{i}(1-z),
$$

obtaining the Rosen-Morse potential,

\section{Schrödinger Operators Reducible to the Rescaled Confluent Equation}

One of the basic exactly solvable equations is the confluent equation, given by the operator

$$
\mathcal{F}\left(a ; c ; z, \partial_{z}\right):=z \partial_{z}^{2}+(c-z) \partial_{z}-a .
$$

It is convenient to consider (4.60) in parallel with the equation given by the operator

$$
\mathcal{F}\left(a, b ;-; z, \partial_{z}\right):=z^{2} \partial_{z}^{2}+(-1+(1+a+b) z) \partial_{z}+a b .
$$

The equation given by $(4.61)$ is sometimes called the ${ }_{2} F_{0}$ equation. Note that

$$
z^{a} \mathcal{F}\left(a, b ;-; z, \partial_{z}\right) z^{-a}=w \mathcal{F}\left(a ; 1+a-b ; w, \partial_{w}\right), \quad w=-z^{-1}, \quad z=-w^{-1} .
$$

Hence the ${ }_{2} F_{0}$ equation is equivalent to the confluent equation. The relationship between the parameters is

$$
c=1+a-b, \quad b=1+a-c .
$$


Another exactly solvable equation that we will consider in this section is sometimes called the ${ }_{0} F_{1}$ equation. It is given by

$$
\mathcal{F}\left(c ; z, \partial_{z}\right):=z \partial_{z}^{2}+(c-z) \partial_{z}-a,
$$

and is equivalent to the Bessel equation.

Clearly, the confluent, ${ }_{2} F_{0}$ and ${ }_{0} F_{1}$ equations belong to the class of hypergeometric type equations.

The basic equation of this section will be (1.5), given by

$$
\mathcal{F}\left(a ; c ; \gamma ; z ; \partial_{z}\right):=z \partial_{z}^{2}+(c-\gamma z) \partial_{z}-a .
$$

It will be called the rescaled confluent equation. Note that in the case $\gamma=1$ (4.63) coincides with the confluent equation. If $\gamma \neq 0,(4.63)$ can be reduced to the confluent equation by rescaling (and hence also to the ${ }_{2} F_{0}$ equation). If $\gamma=0,(4.63)$ coincides with the ${ }_{0} F_{1}$ equation.

The Bose invariant of the rescaled confluent equation equals

$$
{ }_{1} I_{1}(a, b ; \gamma ; z)=\frac{-\gamma^{2} z^{2}+2(c \gamma-2 a) z+2 c-c^{2}}{4 z^{2}} .
$$

\subsection{Symmetries of the Rescaled Confluent Equation}

Let us first describe symmetries of the rescaled confluent equation.

$$
\begin{aligned}
z^{c-1} \mathcal{F}\left(a ; c ; \gamma ; z, \partial_{z}\right) z^{1-c} & =\mathcal{F}\left(a-c \gamma+\gamma ; 2-c ; \gamma ; z, \partial_{z}\right) \\
\mathrm{e}^{-\gamma z} \mathcal{F}\left(a ; c ; \gamma ; z, \partial_{z}\right) \mathrm{e}^{\gamma z} & =-\mathcal{F}\left(c-a ; c ; \gamma ; w, \partial_{w}\right), \quad z=-w
\end{aligned}
$$

Besides, the scaling acts as follows:

$$
\mathcal{F}\left(a ; c ; \gamma ; z, \partial_{z}\right)=\gamma \mathcal{F}\left(a / \gamma ; c ; 1 ; w, \partial_{w}\right), \quad w=\gamma z .
$$

It is convenient to introduce new parameters $\alpha, \nu$ :

$$
\begin{gathered}
a=\frac{\gamma+\alpha \gamma-\nu}{2}, \quad c=1+\alpha ; \\
\alpha=c-1=a-b, \quad \nu=c \gamma-2 a=1-a-b .
\end{gathered}
$$

In the new parameters the Bose invariant of the rescaled confluent equation has a more symmetric form:

$$
{ }_{1} I_{1}(\alpha, \nu, \gamma ; z)=-\frac{\gamma^{2}}{4}+\frac{\nu}{2 z}+\frac{1-\alpha^{2}}{4 z^{2}} .
$$

Thus, the starting point for the further analysis will be the equation

$$
\left(-\partial_{z}^{2}+\frac{\gamma^{2}}{4}-\frac{\nu}{2 z}+\left(\frac{\alpha^{2}}{4}-\frac{1}{4}\right) \frac{1}{z^{2}}\right) \phi(z)=0 .
$$

We will describe three classes of Schrödinger operators solved using the confluent equation corresponding to three obvious choices for $\left(r^{\prime}(z)\right)^{2}$ :

$$
\text { (1) } \frac{1}{4}, \quad \text { (2) } \frac{1}{4 z}, \text { (3) } \frac{1}{4 z^{2}} \text {. }
$$




\subsection{Hydrogen Atom}

We consider (1) of (4.67).We set

$$
z=2 r .
$$

This leads to the Schrödinger equation

$$
\left(-\partial_{r}^{2}+V_{\alpha, \nu}(r)+\gamma^{2}\right) \phi(r)=0,
$$

with the potential

$$
V_{\alpha, \nu}(r):=-\frac{\nu}{r}+\left(\frac{\alpha^{2}}{4}-\frac{1}{4}\right) \frac{1}{r^{2}} .
$$

Equation (4.69) is the radial part of the Schrödinger equation for the hydrogen atom. $] 0, \infty\left[\right.$ is a natural real domain for this equation. If $\nu, \alpha^{2}$ are real, then so is $V_{\alpha, \nu}$ on $] 0, \infty[$.

The derivation of (4.69) from (4.66) is immediate:

$$
\begin{gathered}
4\left(-\partial_{z}^{2}+\frac{\gamma^{2}}{4}-\frac{\nu}{2 z}+\left(\frac{\alpha^{2}}{4}-\frac{1}{4}\right) \frac{1}{z^{2}}\right) \\
=-\partial_{r}^{2}+\gamma^{2}-\frac{\nu}{r}+\left(\frac{\alpha^{2}}{4}-\frac{1}{4}\right) \frac{1}{r^{2}} .
\end{gathered}
$$

\subsection{Rotationally Symmetric Harmonic Oscillator}

We consider (2) of (4.67). We set

$$
z=r^{2}, \quad \text { which solves } z^{\prime}=2 \sqrt{z} \text {. }
$$

This leads to the Schrödinger equation

$$
\left(-\partial_{r}^{2}+V_{\alpha, \gamma}(r)-2 \nu\right) \phi(r)=0,
$$

with the potential

$$
V_{\alpha, \gamma}(r):=\gamma^{2} r^{2}+\left(\alpha^{2}-\frac{1}{4}\right) \frac{1}{r^{2}} .
$$

] $0, \infty\left[\right.$ is a natural real domain. For real $\gamma^{2}, \alpha^{2}$, the potential is real on $] 0, \infty[$. By scaling, the only different real cases are $\gamma^{2}=1,0,-1$.

For $\gamma^{2}=1$ the equation is the radial part of the rotationally symmetric harmonic oscillator. For $\gamma^{2}=0$ the equation is the radial part of the Helmholtz equation.

Here is an explicit derivation of (4.71) from (4.66):

$$
\begin{gathered}
4 z^{1-\frac{1}{4}}\left(-\partial_{z}^{2}+\frac{\gamma^{2}}{4}-\frac{\nu}{2 z}+\left(\frac{\alpha^{2}}{4}-\frac{1}{4}\right) \frac{1}{z^{2}}\right) z^{\frac{1}{4}} \\
=-4 z \partial_{z}^{2}-2 \partial_{z}+\gamma^{2} z-2 \nu+\left(\alpha^{2}-\frac{1}{4}\right) \frac{1}{z} \\
=-\partial_{r}^{2}+\gamma^{2} r^{2}-2 \nu+\left(\alpha^{2}-\frac{1}{4}\right) \frac{1}{r^{2}}
\end{gathered}
$$




\subsection{Morse Potential}

We consider (3) of (4.67). We set

$$
z=\mathrm{e}^{-r}, \quad \text { which solves } z^{\prime}=-z \text {. }
$$

This leads to the Schrödinger equation

$$
\left(-\partial_{r}^{2}+V_{\nu, \gamma}(r)+\frac{\alpha^{2}}{4}\right) \phi(r)=0,
$$

where

$$
V_{\nu, \gamma}(r)=\frac{\gamma^{2}}{4} \mathrm{e}^{-2 r}-\frac{\nu}{2} \mathrm{e}^{-r} .
$$

] $-\infty, \infty\left[\right.$ is a natural real domain. For real $\nu, \gamma^{2}$, the potential is real on ] $-\infty, \infty\left[\right.$. By translation, the only different real cases are $\gamma^{2}=1,0,-1$.

Here is an explicit derivation of (4.73) from (4.66):

$$
\begin{aligned}
z^{2-\frac{1}{2}} & \left(-\partial_{z}^{2}+\frac{\gamma^{2}}{4}-\frac{\nu}{2 z}+\left(\frac{\alpha^{2}}{4}-\frac{1}{4}\right) \frac{1}{z^{2}}\right) z^{\frac{1}{2}} \\
= & -z^{2} \partial_{z}^{2}-z \partial_{z}+\frac{\gamma^{2}}{4} z^{2}-\frac{\nu}{2} z+\frac{\alpha^{2}}{4} \\
& =-\partial_{r}^{2}+\frac{\gamma^{2}}{4} \mathrm{e}^{-2 r}-\frac{\nu}{2} \mathrm{e}^{-r}+\frac{\alpha^{2}}{4} .
\end{aligned}
$$

\section{Schrödinger Equations Reducible to the Translated Harmonic Oscillator}

The last family of exactly solvable Schrödinger equations that we describe is (1.6), given by

$$
-\partial_{z}^{2}+\theta^{2} z^{2}+\rho z+\lambda
$$

We will call (5.74) the translated harmonic oscillator equation. It is already in the canonical form.

If $\theta^{2} \neq 0$, then (5.74) is just the translation of the Schrödinger equation for usual harmonic oscillator. It is then equivalent to the Hermite equation, given by

$$
\mathcal{G}\left(a, y, \partial_{y}\right):=\left(\partial_{y}^{2}-2 y \partial_{y}-2 a\right)
$$

In fact, we have

$$
\begin{aligned}
& \theta \mathrm{e}^{-\frac{y^{2}}{2}} \mathcal{G}\left(a, y, \partial_{y}\right) \mathrm{e}^{\frac{y^{2}}{2}} \\
& \quad=\theta\left(-\partial_{y}^{2}+y^{2}+2 a-1\right) \\
& \quad=-\partial_{z}^{2}+\theta^{2} z^{2}+\rho z+\frac{\rho^{2}}{2 \theta^{2}}+2 a-1,
\end{aligned}
$$

where $y=\sqrt{\theta}\left(z+\frac{\rho}{2 \theta^{2}}\right)$.

For $\theta=0, \rho \neq 0,(5.74)$ is equivalent to the Airy equation

$$
\left(\partial_{y}^{2}+y\right) \psi(y)=0
$$


which in turn is equivalent to a special case of the ${ }_{0} F_{1}$ equation:

$$
\begin{aligned}
& -\partial_{z}^{2}+\rho z+\lambda \\
& \quad=\rho^{\frac{2}{3}}\left(-\partial_{y}^{2}+y\right) \\
& \quad=(\rho 3)^{\frac{2}{3}} w^{\frac{1}{3}} \mathcal{F}\left(\frac{2}{3} ; w, \partial_{w}\right),
\end{aligned}
$$

where we set $y=\rho^{\frac{1}{3}} z+\rho^{-\frac{2}{3}} \lambda$, and $w=3^{-2} y^{3}$.

We will describe three classes of Schrödinger operators that can be reduced to (5.74) corresponding to three obvious choices for $\left(r^{\prime}(z)\right)^{2}$ :

$$
\text { (1) } 1, \quad(2) z, \quad(3) z^{2} \text {. }
$$

\subsection{Translated Harmonic Oscillator}

We consider Ansatz (1) of (5.75), which corresponds to the most obvious choice of the energy, that is $-\lambda$. We rename the variable $z=r$. This leads to the Schrödinger equation

$$
\left(-\partial_{r}^{2}+V_{\theta, \rho}(r)+\lambda\right) \phi(r)=0
$$

where

$$
V_{\theta, \rho}(r)=\theta^{2} r^{2}+\rho r
$$

\subsection{Special Potential I}

We can choose the energy to be $-\rho$, that is Ansatz (2) of (5.75). This corresponds to the substitution

$$
z=\left(\frac{3 r}{2}\right)^{\frac{2}{3}}, \quad \text { which solves } z^{\prime}=z^{-\frac{1}{2}},
$$

and leads to the Schrödinger equation

$$
\left(-\partial_{r}^{2}+V_{\theta, \lambda}(r)+\rho\right) \phi(r)=0,
$$

where

$$
V_{\theta, \lambda}(r)=\theta^{2}\left(\frac{3 r}{2}\right)^{\frac{2}{3}}+\lambda\left(\frac{2}{3 r}\right)^{\frac{2}{3}}-\frac{5}{36} \frac{1}{r^{2}}
$$

In fact,

$$
\begin{aligned}
& z^{\frac{1}{4}-1}\left(-\partial_{z}^{2}+\theta^{2} z^{2}+\rho z+\lambda\right) z^{-\frac{1}{4}} \\
& =-\frac{1}{z} \partial_{z}^{2}+\frac{1}{2 z^{2}} \partial_{z}+\theta^{2} z+\rho+\frac{\lambda}{z}-\left(\frac{1}{4}+\frac{1}{4^{2}}\right) \frac{1}{z^{3}} \\
& =-\partial_{r}^{2}+\theta^{2}\left(\frac{3 r}{2}\right)^{\frac{2}{3}}+\rho+\lambda\left(\frac{2}{3 r}\right)^{\frac{2}{3}}-\left(\frac{1}{4}+\frac{1}{4^{2}}\right)\left(\frac{2}{3 r}\right)^{2} .
\end{aligned}
$$

Note the following intriguing feature of the above potential: the coefficient at $r^{-2}$ is fixed and one cannot change it by rescaling the variable $r$. 


\subsection{Special Potential II}

We can choose the energy to be $-\theta^{2}$, that is Ansatz (3) of (5.75). This corresponds to the substitution

$$
z=(2 r)^{\frac{1}{2}}, \quad \text { which solves } z^{\prime}=z^{-1},
$$

and leads to the Schrödinger equation

$$
\left(-\partial_{r}^{2}+V_{\rho, \lambda}(r)+\theta^{2}\right) \phi(r)=0
$$

where

$$
V_{\rho, \lambda}(r)=\frac{\rho}{(2 r)^{\frac{1}{2}}}+\frac{\lambda}{2 r}-\frac{3}{16} \frac{1}{r^{2}}
$$

In fact,

$$
\begin{aligned}
& z^{\frac{1}{2}-2}\left(-\partial_{z}^{2}+\theta^{2} z^{2}+\rho z+\lambda\right) z^{-\frac{1}{2}} \\
& =-\frac{1}{z^{2}} \partial_{z}^{2}+\frac{1}{z^{3}} \partial_{z}+\theta^{2}+\frac{\rho}{z}+\frac{\lambda}{z^{2}}-\left(\frac{1}{2}+\frac{1}{2^{2}}\right) \frac{1}{z^{4}} \\
& =-\partial_{r}^{2}+\theta^{2}+\frac{\rho}{(2 r)^{\frac{1}{2}}}+\frac{\lambda}{2 r}-\left(\frac{1}{2}+\frac{1}{2^{2}}\right) \frac{1}{2^{2} r^{2}}
\end{aligned}
$$

Again, the coefficient at $r^{-2}$ is fixed and one cannot change it by rescaling the variable $r$.

\section{Acknowledgements}

The research of J. D. was supported in part by the grant N N201 270135 of the Polish Ministry of Science and Higher Education.

Open Access. This article is distributed under the terms of the Creative Commons Attribution Noncommercial License which permits any noncommercial use, distribution, and reproduction in any medium, provided the original author(s) and source are credited.

\section{References}

[1] Bose, A.K.: A class of solvable potentials. Nuov. Cim. 32, 679 (1964)

[2] Cayley, A.: On the Schwarzian derivative and the polyhedral functions. Trans. Camb. Phil. Soc. 13, 5-68 (1880)

[3] Cooper, F., Khare, A., Sukhatme, U.: Supersymmetry in Quantum Mechanics. World Scientific, Singapore (2001)

[4] Cotfas, N.: Shape invariant hypergeometric type operators with application to quantum mechanics. Cent. Eur. J. Phys. 4, 318-330 (2006)

[5] Eckart, C.: The Phys. Rev. 35, 1303-1309 (1930)

[6] Flügge, S.: Practical Quantum Mechanics. Springer, Berlin (1971)

[7] Ginocchio, J.N.: A class of exactly solvable potentials. I. One-dimensional Schrödinger equation. Ann. Phys. 152, 203-219 (1984) 
[8] Hull, T.E., Infeld, L.: The factorization method. Rev. Mod. Phys. 23, 21-68 (1951)

[9] Hulthén, L.: Über die Eigenlösungen der Schrödinger-Gleichung der Deuterons. Ark. Mat. Astron. Fys. A 28(5), 1-12 (1942)

[10] Kummer, E.: Über die hypergeometrische Reihe. Crelle 15 39-83, 127-172 (1836)

[11] Khare, A., Sukhatme, U.: New shape-invariant potentials in supersymmetric quantum mechanics. J. Phys. A 26, 901-904 (1993)

[12] Liouville, J.: Sur le développement des fonctions ou parties de fonctions en séries dont les divers termes sont assujettis à satisfaire à une même équation différentielle du second ordre contenant un paramètre variable. J. Math. Pure Appl. 2, 16-35 (1837)

[13] Milson, R.: On the Liouville transformation and exactly-solvable Schrödinger equations. Int. J. Theor. Phys. 37, 1735-1752 (1998)

[14] Morse, P.M.: Diatomic molecules according to the wave mechanics. II. Vibrational levels. Phys. Rev. 34, 57-64 (1929)

[15] Manning, M.F., Rosen, N.: A potential function for the vibrations of diatomic molecules. Phys. Rev. 44, 953 (1933)

[16] Natanzon, G.A.: Study of the one dimensional Schrödinger equation generated from the hypergeometric equation. Vestnik Leningrad Univ. 10, 22-28 (1971)

[17] Nikiforov, A.F., Uvarov, V.B.: Special Functions of Mathematical Physics. Birkhäuser, Basel (1988)

[18] Osgood, B: Old and New on the Schwarzian derivative. Quasiconformal Mappings and Analysis, pp. 275-308. Springer, New York (1998)

[19] Pöschl, G., Teller, E.: Bemerkungen zur Quantenmechanik des anharmonischen Oszillators. Z. Phys. 83(3-4), 143-151 (1933)

[20] Scarf, F.: New soluble energy band problem. Phys. Rev. 112, 1137-1140 (1958)

[21] Schwarz, H.: Über diejenigen Fälle, in welchen die Gaussische hypergeometrische Reihe eine algebraische Function ihres vierten Elementes darstellt. J. Math. 75, 292-335 (1873)

[22] Schrödinger E.: A method of determining quantum-mechanical eigenvalues and eigenfunctions. Proc. R. Irish Acad. A 46, 9-16 (1940)

[23] Saxon, D.S., Woods, R.D.: Diffuse surface optical model for nucleon-nuclei scattering. Phys. Rev. 95, 577-578 (1954)

[24] Whittaker, E.T., Watson, G.N.: A Course of Modern Analysis, vols. I, II. 4th edn (reprint of the 1927 edition). Cambridge University Press, New York (1962)

\author{
Jan Dereziński \\ Department of Mathematical Methods in Physics \\ Faculty of Physics \\ University of Warsaw \\ Hoża 74 \\ 00-682 Warsaw \\ Poland \\ e-mail: jan.derezinski@fuw.edu.pl
}


Michał Wrochna

RTG "Mathematical Structures in Modern Quantum Physics"

Institute of Mathematics

University of Göttingen

Bunsenstr. 3-5

37073 Göttingen

Germany

e-mail: wrochna@uni-math.gwdg.de

Communicated by Claude-Alain Pillet.

Received: September 26, 2010.

Accepted: November 15, 2010. 